\title{
In silico mutational analysis in RNA polymerase $\beta$ subunit (rpoB) gene of rifampicin-resistant in Mycobacterium tuberculosis from Malaysia
}

\author{
Ernie Zuraida Alia ${ }^{{ }^{*}}$, Nurul Hamizah Hamidon ${ }^{\mathrm{b}}$, Rahizan Issa ${ }^{\mathrm{b}}$ \\ "Inborn Error of Metabolism and Genetic Unit, Nutrition, Metabolism and Cardiovascular Research Centre, Institute for Medical Research, \\ National Institutes of Health, Ministry of Health Malaysia, Selangor, Malaysia \\ ${ }^{b}$ Bacteriology Unit, Infectious Disease Research Centre, Institute for Medical Research, National Institutes of Health, Ministry of Health \\ Malaysia, Selangor, Malaysia
}

Received 11th February 2021 / Accepted 4th July 2021

\begin{abstract}
Tuberculosis (TB) is mainly caused by Mycobacterium tuberculosis (MTB) and remains as a key public health problem worldwide. Most of MTB clinical strains are resistant to rifampicin (RIF), the firstline anti-tuberculosis drug. RIF resistance to MTB is due to mutations that mainly found in RIF resistancedetermining region (RRDR) in drug target gene, RNA polymerase $\beta$ subunit $(r p o B)$. Therefore, the aim of the study is to extend the identification of variants in $r p o B$ gene and to elucidate the effect of variants to the RIF resistance. Four of the strains, MTBR1/09, MTBR2/09, MTBR3/09 and MTB221/11 were subjected to drug susceptibility test (DST). All of the strains sequenced and submitted to the National Center for Biotechnology Information Sequence Read Archive were analyzed to identify the variants in the ${ }_{p o} B \mathrm{~B}$ gene. The identified new variants were then subjected to docking to examine the drug-protein interactions. DST analysis revealed MTBR1/09, MTBR2/09 and MTBR3/09 were resistant to the RIF drug, while MTB221/11 was a presumptive susceptible strain. Two new variants were observed, the deletion (Phe433_Met434delinsLeu in MTBR1/09) and missense (Lys37Arg in MTBR3/09) variants. Meanwhile, the His445Leu, Ser450Leu, Asp103Asp, Ala1075Ala were reported variants. Docking of RIF to MTBR1/09 and MTBR3/09 mutant models revealed the RIF bound to the RIF binding site at different binding affinity and conformation. Concurrently, the new variants caused the RIF to bind to the different active site and neighboring residues. Findings from DST and docking analyses indicate that new variants potentially disturb the RIF inhibition in RpoB mutant proteins, and thus might be responsible to cause the RIF resistance.
\end{abstract}

Keywords: molecular docking, Mycobacterium tuberculosis, rifampicin, RNA polymerase $\beta$ subunit gene, variant

\section{INTRODUCTION}

Tuberculosis (TB) is the ninth leading cause of mortality worldwide. In 2018, an estimated 10 million people have been reported to be infected with TB by World of Health Organization (WHO) (World Health Organization, 2019). The recent enhancement in multi-drug resistant (MDR-TB) and extensively drug resistant (XDRTB) has worsen the situation. Both situations are predominantly granted by the accumulation of mutations in drug target genes such as $r p o B, p n c A$, kat G, gyrA and gyrB of MTB (Zhang et al., 2013; Singh et al., 2017).

Mutations in $r p o B$ gene can be discovered in 96\% of RIF-resistant MTB strains worldwide (Gerald et al., 2014). RIF is one of the important chemotherapeutic agents in the TB treatment

*Author for correspondence: Ernie Zuraida Ali, Inborn Error of Metabolism, Nutrition and Cardiovascular Research Centre, Institute for Medical Research, National Institutes of Health, Ministry of Health Malaysia, Selangor, Malaysia. Email - erniez1980@gmail.com / ernie@moh.gov.my 
(Cole, 1996; Campbell et al., 2001; Kumar and Lingaraja, 2014). RIF target is the RNA polymerase (RNAP) enzyme (Zhang et al., 1999; Campbell et al., 2001; Borukhov \& Nudler, 2003; Singh et al., 2017). RNAP is an important enzyme in transcription process and it consists of five different subunits, such as $\alpha$-dimer, $\beta$ subunit, $\beta$ ' subunit and $\omega$ subunit (Zhang et al., 1999; Campbell et al., 2001; Nusrath et al., 2016). RNAP is the last target for regulatory pathways that regulate gene expression in all living organisms (Zhang et al., 1999; Campbell et al., 2001; Nusrath et al., 2016). RIF binds to the $\beta$-subunit of the RNAP encoded by the $r p o B$ gene and consequently, inhibits the elongation of messenger RNA (Campbell et al., 2001; Almeida da Silva and Palomino, 2011; Singh et al., 2017). In $r p o B$ gene, the RIF resistance mostly occurs due to mutation within an 81 base pair (bp) hot spot region or known as RRDR (Almeida da Silva and Palomino, 2011; Pang et al., 2013; Zaw et al., 2018; Al-Mutairi et al., 2019). Previous studies also revealed RIF resistance to the mutations outside of the RRDR (Almeida da Silva and Palomino, 2011; Pang et al., 2013; Singh et al., 2017). Majority of the identified mutations are non-synonymous mutations (Sharma and Madan, 2014; Zaw et al., 2018). However, other changes can also be correlated to the RIF resistance such as insertion, deletion and frameshift (Mani et al., 2001; Sharma and Madan, 2014).

In 2017, there were 26,422 patients in Malaysia diagnosed with TB, with the incidence rate is $81.30 / 100,000$ populations (Fakhruzzaman et al., 2019). In addition, several genome studies have also been carried out to MTB local isolates (Philip et al., 2016; Issa et al., 2016; Fakhruzzaman et al., 2019; Jani et al., 2019). Most of the identified variants from the previous studies are listed in the TB profiler (Fakhruzzaman et al., 2019; Jani et al., 2019). Besides that, in silico studies have also been performed to predict the effect of mutations in order to characterize and understand the association between the clinical mutants in RpoB protein and RIF, related to the resistance (Nusrath et al., 2016; Nusrath \& Hanna, 2017; Prakash et al., 2017). Therefore, this study was performed to extend the identification of variants spectrum for the most important drug target $r p o B$ gene of MTB in Malaysia isolates. Furthermore, structures of the variants along with molecular docking studies were undertaken to explore the effect these variants to the drug resistance. Finding from this study will provide better understanding to the role of the variants in the mechanism of RIF resistance.

\section{MATERIALS AND METHODS}

\section{Identification of variants}

The MTBR1/09, MTBR2/09 and MTBR3/09 strains were isolated from pulmonary samples, whereas MTB221/11 was isolated from cerebrospinal fluids (CSF) in Malaysia patients. This study used bacterial isolates, thus, no formal ethics approval was required. Drug susceptibility testing (DST) was performed to all strains with first-line anti-TB drug, namely rifampicin (RIF). All strains have been subjected to whole-genome shotgun sequencing, using Illumina MiSeq platform. The CLC Genome Workbench Software version 6.0.1 was used to filter and analyze the raw sequence data. The sequenced data have also been deposited in the National Center for Biotechnology Information Sequence Read Archive (BioProject ID PRJNA272470) with the first version accession numbers: LFLI00000000 (MTB MTB221/11), LATN00000000 (MTB MTBR1/09), LATO00000000 (MTB MTBR2/09) and LATP00000000 (MTB MTBR3/09).

From the submitted sequences, the drug target gene, $r p o B$, was selected, analyzed and confirmed for their identity using MEGA7 software (version 7), aligning sequences with the MTB reference (Kumar et al., 2016). The nucleotide sequences were converted to amino acids by using Expasy Translate (http://web.expasy.org/cgi-bin/translate/dna aa) and also referred to the MTB reference using Clustal Omega in order to identify the variants (Sievers et al., 2011). Each variant was compared and validated with that listed in Genome-wide MTB variation (GMTV) database (Chernyaeva et al., 2014) and other available reports of drug resistant MTB genotypes in order to identify either the variant is new or reported.

\section{Molecular modelling of rpoB protein}


The three-dimensional (3D) wild type crystal structure of MTB RNA Polymerase beta subunit (RpoB) has been crystallized and available in the Protein Data Bank (PDB) (PDB ID: 5UH6, 5UHB, 5UHC, 5UHD AND 5UHG) (Lin et al., 2017). The crystal structure was visualized using the PyMOL software (version 2.3) (DeLano, 2002). The mutant RpoB protein model was developed according to the user template method using ProMod3 (version 1.3.0), under SWISSMODEL, a fully automated protein structure homology-modelling server (Waterhouse et al., 2018). The PDB ID: 5UH6 coordinates were used as a template as it contains RNA polymerase (RNAP), nucleic acid scaffold with DNA, three nucleotides of RNA complementary to the template DNA strand and RIF.

The small structure alterations, or clashes occurred during the modelling process were resolved by energy minimization. The server uses CHARMM27 force field for parameterization (Mackerell et al., 2004). QMEAN scoring function was used to evaluate the global and local model quality (Benkert et al., 2011). Good model should present QMEAN Z-scores around zero, which represents the model-built structure and experimental structure in similar size. Meanwhile, scores of -4.0 or below indicates the model structure in low quality (Benkert et al., 2011).

\section{Mutant models validation}

The mutant models were then evaluated with ProSA (Wiederstein and Sippl, 2007), which uses the molecular mechanics force field. The overall model quality was predicted by using $\mathrm{z}$-score. PROCHECK (Laskowski et al., 2012) produced the Ramachandran plot statistics for the anticipated model according to stereochemical quality and accuracy, which indicate the acceptance model should have residues located within the accepted regions to be more than $90 \%$. ERRAT (Colovos and Yeates, 1993) was then used mostly to check the quality of non-bonded atomic interactions. The model must have score to be above than $50 \%$, indicating as a good quality model.

\section{Preparation of ligand}

For the ligand, the PubChem database was used to retrieve the 3D structure of rifampicin (RIF) (PubChem ID: 135398735) (Bolton et al., 2008). Open Babel was then used to transform the ligand from the SDF format to the PDB format (Guha et al., 2006).

\section{Molecular docking}

Initially, redocking was performed in order to confirm the structure and acquire the docking standard parameter. The RIF was redocked to the wild type of RpoB monomer structure. The optimized parameters (exhaustiveness and number of runs) were examined according to the wild type structure to acquire the highest binding affinity and similar RIF conformation.

Initial files for protein and ligand based on PQR and PDB formats, respectively were constructed by AutoDock Tools 1.5.6 (ADT). The two files were transformed to PDBQT, which would be applicable in AutoDock Vina (Sanner, 1999; Trott and Olson, 2010). The grid point spacing of $1.0 \AA$ was used before adjusting the grid box, as that is the criterion unit in AutoDock Vina. The grid box was assigned at 24 $\AA \times 24 \AA \times 24 \AA$, centered at the $\mathrm{x}, \mathrm{y}$, and $\mathrm{z}$ ($12.988,0.913,21.035)$ coordinates, which was to cover all the binding site residues that recognized to interact with the RIF and involved in the catalytic activity; based on the literature (Sekhar et al., 2008). Consequently, docking calculation was performed to both protein and ligand files by AutoDock Vina. The default settings were used for all other parameters. The protein was retained rigid and the ligand was permitted to vary.

The finding was assessed using the binding affinity score in $\mathrm{kcal} / \mathrm{mol}$. Classification based on the lowest docked energy would determine the best ligand conformation. The RIF ligands in mutant and wild type structures were overlaid to check the conformation of both ligands. The ligand binding modes, the root mean standard deviation (RMSD) and hydrogen-bond interactions between the protein and the ligand were examined using PyMol (DeLano, 2002; Peterson et al., 2014). 

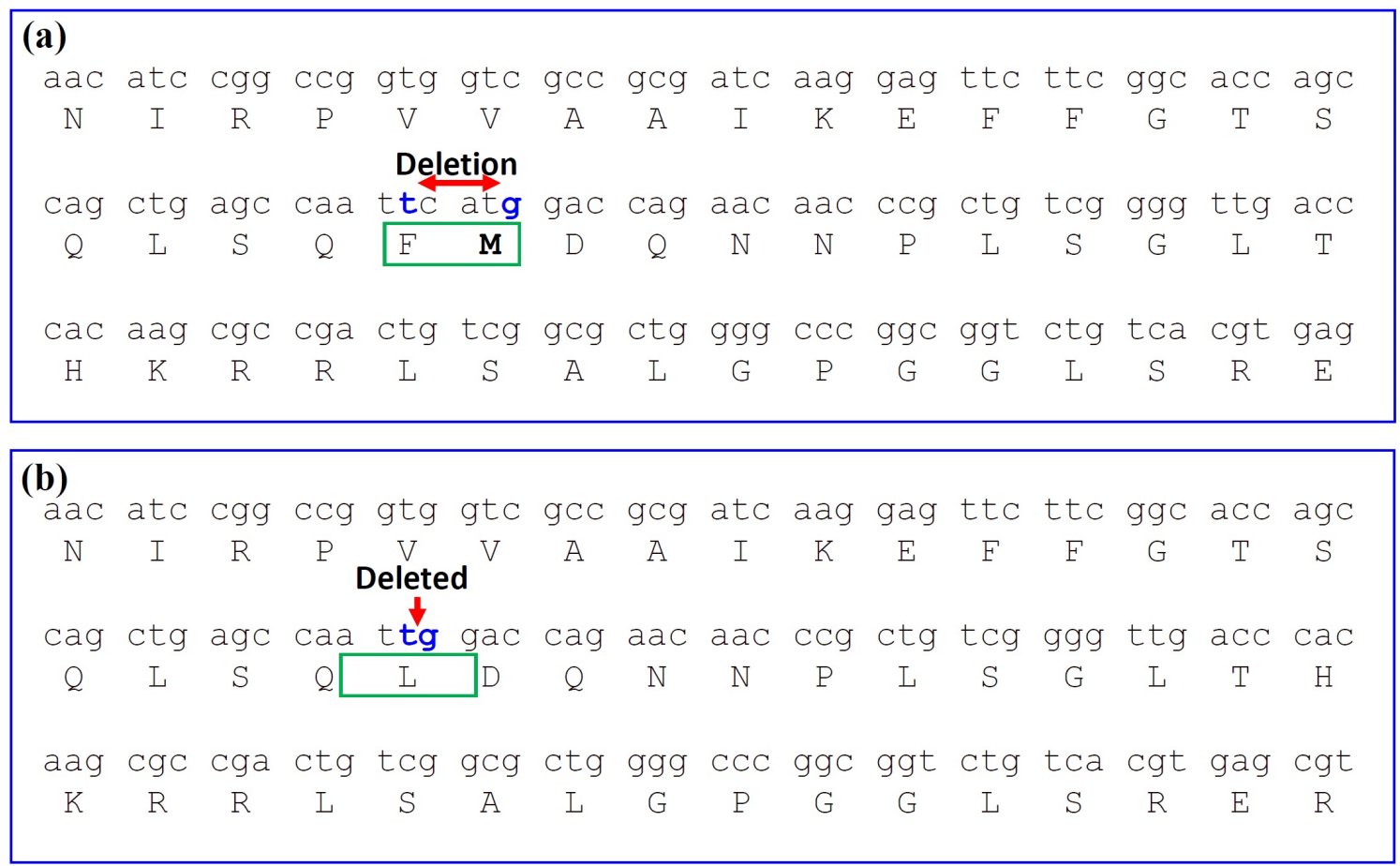

Figure 1. Deletion of c.1299_1301delCAT in MTBR1 strain. (a) Deletion of nucleotide CAT alters the wild type amino acid by deleting two amino acids (Phe and Met). (b) Deletion of amino acids Phe and Met is replaced by only one amino acid, Leu.

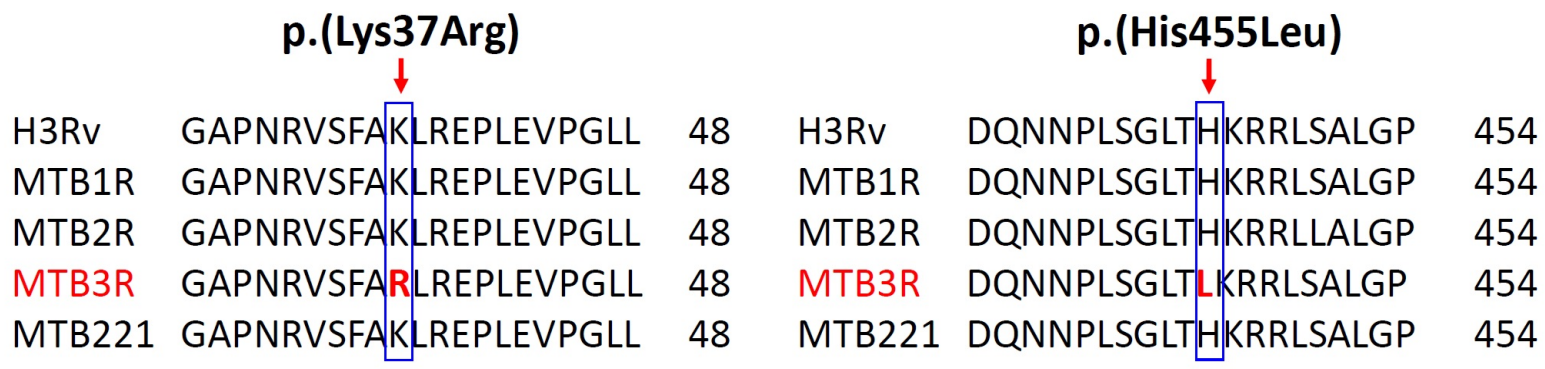

Figure 2. Multiple sequence alignment of $r p o B$ gene for four isolates from Malaysia. Changes in amino acids at three positions are shown in blue box.

\section{RESULTS}

\section{Variants in rpoB gene}

The MTBR1/09, MTBR2/09 and MTBR3/09 were phenotype resistant to RIF drug. Meanwhile, one strain, MTB221/11 was a presumptive susceptible strain. Sequencing analyses of $r p o B$ gene were performed for identification of variants. One new deletion variant, three nonsynonymous variants and two synonymous variants were identified. A new deletion, c.1299_1301delCAT was identified in MTBR1/09 strain. Deletion of nucleotide CAT caused deletion of two amino acids (Phe and Met), replaced with amino acid, Leu, 
(p.(Phe433_Met434delinsLeu) as shown in Figure 1.

Multiple sequence alignment of $r p o B$ gene revealed sequence variations in different strains at the amino acid level for three non-synonymous variants (Figure 2). One new missense variant, c.110A $>$ G; p.(Lys37Arg) and one reported missense variant, c.1334A $>$ T; p.(His445Leu) were detected in MTBR3/09 strain. Another missense variant, c.1349C $>$ T; p.(Ser450Leu) was detected in MTBR2/09 strain. Meanwhile, silent variants, c.309C $>\mathrm{T}$; p.(Asp103Asp) was detected in MTB221/09 and c.3225T>C; p.(Ala1075Ala) was identified in MTBR1/09, MTBR2/09 and MTBR3/09. Three variants were located within the RRDR (Phe433_Met434delinsLeu, His445Leu and Ser450Leu), which was near to the RIF binding site, while the other variants located outside the RRDR (Lys37Arg, Asp103Asp and Ala1075Ala).

\section{Mutant modelling}

To address the effect of new variants to the RpoB protein structure and to observe how these new variants cause the RIF resistance, homologymodelling server was used to generate the mutant models. The X-ray crystal structure, PDB ID: $5 \mathrm{UH} 6$ was used as RpoB wild type reference and template to generate the mutant models. A sequence of MTBR1/09 strain was used to develop a mutant model for Phe433_Met434delinsLeu, while sequence of MTBR3/09 strain was used to develop a mutant model for Lys37Arg and His445Leu. Table 1 shows the two modelled structures were acceptable as all of them have more than 99\% similar sequence with the reference template (PDB ID: 5UH6). The two-modelled structures also showed that the QMEAN Z-scores around zero, indicating the model structures and template structure are in similar size.

\section{Validation of the rpoB mutant model}

The optimized new mutant structures were validated using ProSA-web and PROCHECK programs. ProSA-web was applied to inspect the erroneous of 3D mutant models. The Z-score, which denoted the model quality and random conformation, was used to determine the difference of the total energy of the structure with respect to an energy distribution. The ProSA-web analysis presented the Z-scores of MTBR1 and MTBR3 mutant models were -14.48 and -14.96 respectively, indicating there was no major deviation from wild type structure of a similar size of the target protein's Z-score, which was -14.59 (Table 1).

PROCHECK was used to further inspect the stereochemistry quality and structure feature. For a better-quality model, the residues positioned in the most favorable and additional allowed regions must be more than $90 \%$. Table 1 shows the scores of wild type and mutant models produced by the PROCHECK were in good quality. The PROCHECK result showed that the $87.6 \%$ and $12.2 \%$ of wild type residues were located in favored core and in additional allowed regions. Meanwhile, in the mutant models, $92.0 \%$ and $7.5 \%$ of MTBR1 and, $92.1 \%$ and $7.8 \%$ of MTB3R residues were located in favored core and in additional allowed regions. As both mutant models showed a good quality, hence, these model structures were used for docking with the RIF inhibitor.

\section{Docking of RIF in RpoB wild type and new variant structures}

Molecular docking was performed to observe how the new variants cause RIF resistance. The docked complexes were analyzed and PyMOL was used to observe the interactions between RIF and $\mathrm{RpoB}$ wild type as well as RpoB mutant proteins. The docking of RIF with RpoB wild type and mutant proteins exhibited the change of binding energies and hydrogen bonds pattern.

Initially, redocking was performed to the wild type structure (RIF and RpoB monomer structure (PDB ID: 5UH6)). The results of redocking infer that the docked of RIF to the RpoB monomer structure bound at the same binding site of wild type X-ray crystal structure. The docked RIF to the RpoB monomer structure produced binding affinity score of $-10.2 \mathrm{kcal} / \mathrm{mol}$ (Table 2). Superposition of the docked RIF from the wild type structure and RIF from the X-ray crystal structure produced an RMSD of $0.06 \AA$, yielded a good agreement (Figure 3a). Structural observation showed the docked RIF embedded between 4 active site residues (Gln438, Phe439, Arg454 and Ser456) in the wild type RpoB ligandbinding site, similar to X-ray crystal structure (Table 2). Besides that, the redocked RIF also 
formed a new hydrogen bond with Arg173, which previously was not found in the X-ray crystal structure as described in Figure 4a.

Docking of RIF to the MTBR1/09 mutant model produced higher value of binding affinity score of $-11.7 \mathrm{kcal} / \mathrm{mol}$ than wild type (Table 2). However, superposition between RIF from the mutant model and RIF from wild type structures produced an RMSD of $0.12 \AA$ and exhibited that the deletion of Phe and Met in codon 433 to 434 caused the displacement of RIF (Figure 3b). The RIF was observed to lose hydrogen bond interactions with the active site residues, $\operatorname{Arg} 173$, Gln438, Phe439, Arg454 and Ser456 (Figure 4b). Concurrently, the RIF was associated with hydrogen bonds, His1029 (NE2) and Arg67 (NH1) (Figure 4b). This variant also was observed to form a new interaction with neighboring residue, Asn671 (ND2) (Figure 5a).

Meanwhile, docking of RIF to the MTB3R/09 mutant model produced similar binding affinity score $(-10.5 \mathrm{kcal} / \mathrm{mol})$ with wild type (Table 2). Superposition between RIF from mutant model and RIF from wild type produced an RMSD of $0.09 \AA$ and exhibited variants, $A r g 37$ and Leu445 to affect the RIF conformation (Figure 3c). However, these variants were observed not to affect the hydrogen bond interactions with all the active site residues (Arg173, Gln438, Phe439, Arg454 and Ser456) (Figure 4c). Concurrently, the new variant, Lys37Arg formed a new hydrogen bond interaction with neighboring residue, Ser540 (O), while reported variant, His445Leu caused loss of hydrogen bond interactions with neighboring residues, Asp435 (N) and Gln432 (OE1) (Figure $5 b)$.

\section{DISCUSSION}

The molecular basis of multi-drug resistance in treating tuberculosis has well been examined (Prakash et al., 2017; Fakhruzzaman et al., 2019). Most MTB clinical strains are resistant to one of the first-line drugs, which is RIF (Cole, 1996; Kumar and Lingaraja, 2014; Goldstein, 2014). Earlier studies indicate the major cause of RIF resistance is due to mutations that occur in the most important drug target gene, $r p o B$ (Almeida da Silva and Palomino, 2011). The majority of the mutations are known to alter the nature of the MTB mechanism (Zaw et al., 2018; Al-Mutairi et al., 2019). In the present study, the sequencing analysis of $r p o B$ gene from four strains, MTBR1/09 MTBR2/09, MTBR3/09 and MTBR211/09, isolated from Malaysia patients identified the new deletion and missense variants, as well as five reported variants. To the best of our knowledge, the deletion of Phe433_Met434delinsLeu and Lys37Arg identified from MTBR1/09 and MTBR3/09 strains, respectively, were the first variants found in the $r p o B$ gene. In addition, the DST revealed the new variants from the two strains were resistant to RIF.

Previous structural studies on the drug resistance of RIF in various mutations of MTB isolate lead to the impairment of the stability and flexibility of RpoB protein binding site (Kumar and Lingaraja, 2014; Prakash et al., 2017). Therefore, docking study was performed to these two new variants in order to investigate how these variants affect the mechanism of RIF resistance. The deletion of Phe433_Met434delinsLeu caused the structural change on MTBR1/09 mutant model due to the deletion of two amino acids and replaced by one amino acid, as compared to the wild type structure. The docking analysis revealed this structural change led to a different orientation and positioning of RIF in the RIF binding site. Concurrently, the higher docking value resulted from the docking of RIF towards the MTBR1/09 mutant model indicates the high capability of RIF to bind to the RIF binding site. However, based on the hydrogen bond interactions analysis and with the consideration that the amino acid deletion occurred close to the active site, RIF was observed to bind to different residues in MTBR1/09 mutant model than the proposed active site residues. As known, hydrogen bonds are important in the structure stability and also in function of biology molecules especially in enzyme catalysis (De Azevedo Jr., 2010). Instead of RIF forming hydrogen bond interactions with 5 active site residues in the wild type structure, the RIF was able to form only 2 hydrogen bonds with the MTBR1/09 mutant model, which might be attributed by the amino acid deletion in the mutant. RIF bound to the MTBR1/09 mutant model with high affinity is probably influenced by 
another interaction such as electrostatic force and also stable hydrogen bond. Although the high docking score indicates the high affinity, the deletion changed the nature of RIF binding site and altered the interactions of RIF with the active site residues, and therefore may contribute to the RIF resistance in this MTBR1/09 mutant protein.

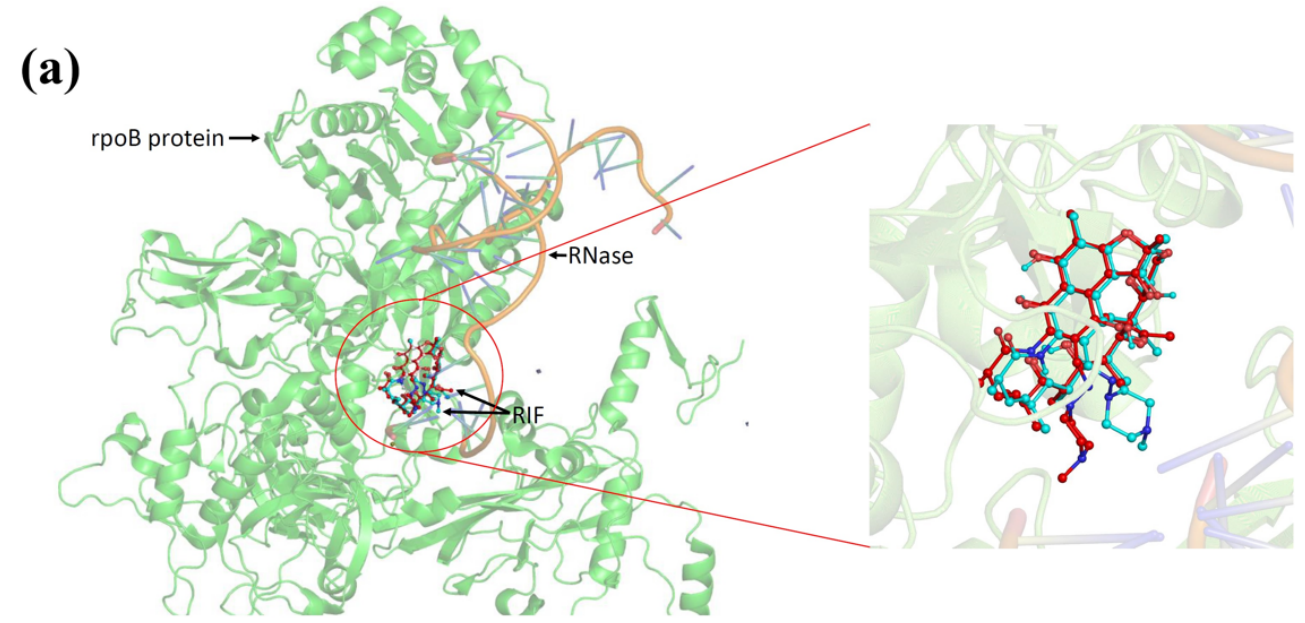

(b)

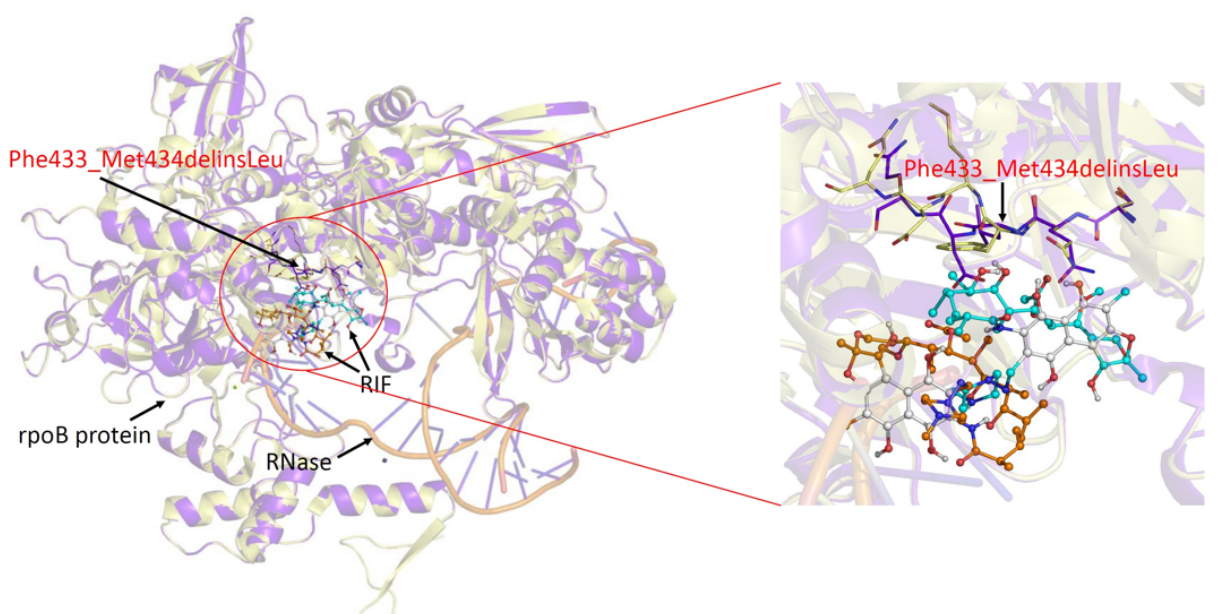

(c)

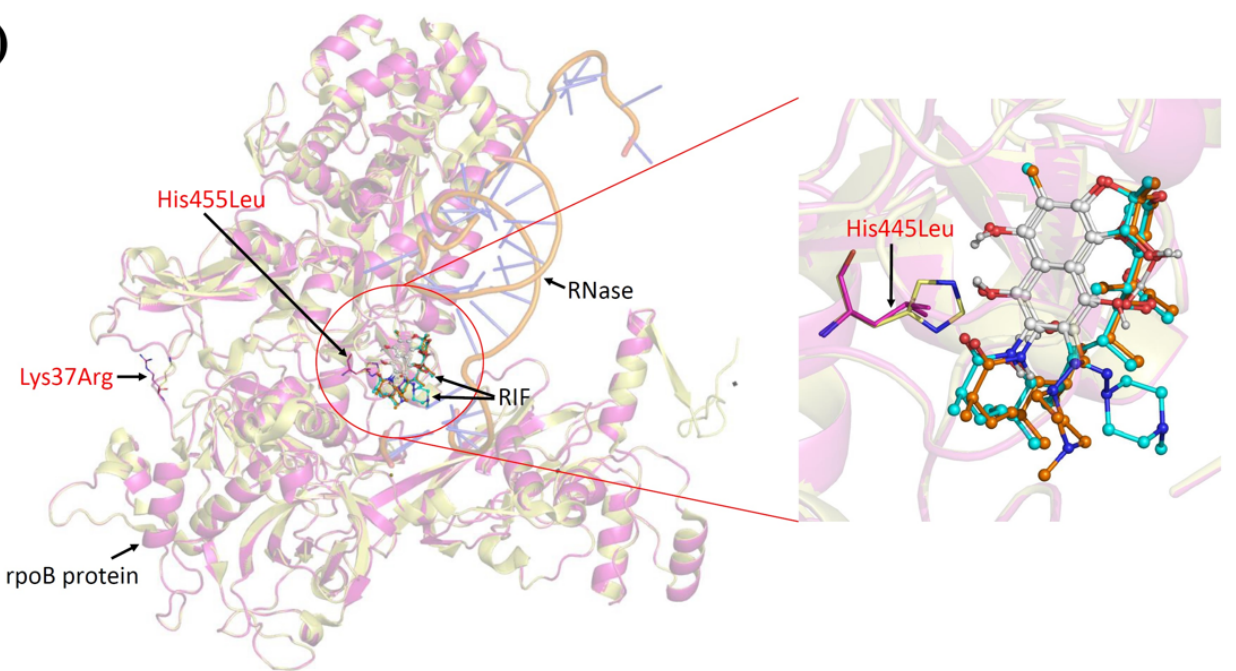

Figure 3. Superimposition of RIF inhibitor in wild type and mutant RpoB structures. (a) Wild type rpoB structure. (b) Deletion of variant, Phe433_Met434delinsLeu in MTBR1/09 strain. (c) Missense variants, Lys37Arg and His445Leu in MTBR3/09 strain. RIF inhibitor in wild type from PDB is in red, RIF inhibitor in wild type from re-docking is in cyan and RIF inhibitor in mutant is in orange. Wild type residues are in yellow. Mutant residues are in purple and magenta. 
Table 1. Sequence identity, QMEAN and quality structures of wild type and new mutant models.

\begin{tabular}{|c|c|c|c|c|c|c|c|c|}
\hline \multirow[b]{2}{*}{ Strain } & \multirow[b]{2}{*}{ Residue } & \multirow[b]{2}{*}{$\begin{array}{c}\text { Sequence } \\
\text { identity } \\
(\%)\end{array}$} & \multirow[b]{2}{*}{$\begin{array}{c}\text { QMEAN } \\
(\mathrm{Z} \text {-score)* }\end{array}$} & \multirow{2}{*}{$\begin{array}{c}\text { ProSA-web } \\
\text { Z-score }\end{array}$} & \multicolumn{4}{|c|}{ ProCheck } \\
\hline & & & & & $\begin{array}{c}\text { Most } \\
\text { favoured } \\
\text { regions } \\
(\%)\end{array}$ & $\begin{array}{c}\text { Additional } \\
\text { allowed } \\
\text { regions } \\
(\%)\end{array}$ & $\begin{array}{c}\text { Generously } \\
\text { allowed } \\
\text { regions } \\
(\%)\end{array}$ & $\begin{array}{c}\text { Disallowed } \\
\text { region } \\
(\%)\end{array}$ \\
\hline Wild Type & $\mathrm{NA}$ & NA & $\mathrm{NA}$ & -14.59 & 87.6 & 12.2 & 0.2 & 0.0 \\
\hline MTBR1 & Phe433_Met434delinsLeu & 99.27 & -0.13 & -14.48 & 92.0 & 7.5 & 0.3 & 0.1 \\
\hline MTBR3 & Lys37Arg and His445Leu & 99.82 & -0.26 & -14.96 & 92.1 & 7.8 & 0.1 & 0.0 \\
\hline
\end{tabular}

Note: ${ }^{*}$ Z-scores $=\sim 0$, indicating the model structure and template structure are in similar size; Z-scores $=-4.0$ or below indicates the model structure in low quality.

Table 2. Binding affinity and hydrogen bond interactions between RFP and neighboring residues, between mutant residues and neighboring residues of native and new mutant models.

\begin{tabular}{|c|c|c|c|c|c|}
\hline \multirow[b]{2}{*}{ Strain } & \multirow[b]{2}{*}{ Residue } & \multirow{2}{*}{$\begin{array}{c}\text { Binding } \\
\text { affinity } \\
\text { (kcal/mol) }\end{array}$} & \multirow{2}{*}{$\begin{array}{c}\text { Residue } \\
\text { interact with } \\
\text { RFP at ligand } \\
\text { binding } \\
\text { pocket }\end{array}$} & $\begin{array}{l}\text { Wild type residue interact } \\
\text { with neighboring residue }\end{array}$ & $\begin{array}{c}\text { Mutant residue interact with } \\
\text { neighboring residue }\end{array}$ \\
\hline & & & & $\begin{array}{c}\text { Wild type residue (Atom): } \\
\text { Neighboring residue } \\
\text { (Atom) }\end{array}$ & $\begin{array}{c}\text { Mutant residue (Atom): Neighboring } \\
\text { residue (Atom) }\end{array}$ \\
\hline $\begin{array}{l}\text { Wild } \\
\text { Type }\end{array}$ & NA & -10.2 & $\begin{array}{c}\text { Arg173, } \\
\text { Gln438, } \\
\text { Phe439, } \\
\text { Arg454, Ser456 }\end{array}$ & -NA- & -NA- \\
\hline MTB1R & Phe433_Met434delinsLeu & -11.7 & $\begin{array}{l}\text { His1029, } \\
\text { Arg607 }\end{array}$ & $\begin{array}{c}\text { Met434(N):Glu171(O) } \\
\text { Asn679(ND2):Met434(O) }\end{array}$ & Leu433(O):Asn671(ND2) \\
\hline \multirow{2}{*}{ MTB3R } & Lys37Arg & -10.5 & $\begin{array}{c}\text { Arg173, } \\
\text { Gln438, } \\
\text { Phe439, } \\
\text { Arg454, Ser456 }\end{array}$ & $\begin{array}{l}\text { Lys37(N):Asp972(OD1) } \\
\text { Lys37(NZ):Ala538(O) }\end{array}$ & $\begin{array}{l}\text { Arg37(N):Asp972(OD1) } \\
\text { Arg37(N):Asp972(OD2) } \\
\text { Arg37(NH1):Ser540(O) } \\
\text { Arg37(NH2):Ser540(O) }\end{array}$ \\
\hline & His445Leu & & & $\begin{array}{c}\text { Asp435(N):His445(ND1) } \\
\text { His445(N):Ser441(O) } \\
\text { His445(NE2):Gln432(OE1) } \\
\text { Arg448(N):His445(O) }\end{array}$ & $\begin{array}{l}\text { Leu445(N):Ser441(O) } \\
\text { Leu445(O):Arg448(N) }\end{array}$ \\
\hline
\end{tabular}


(a)

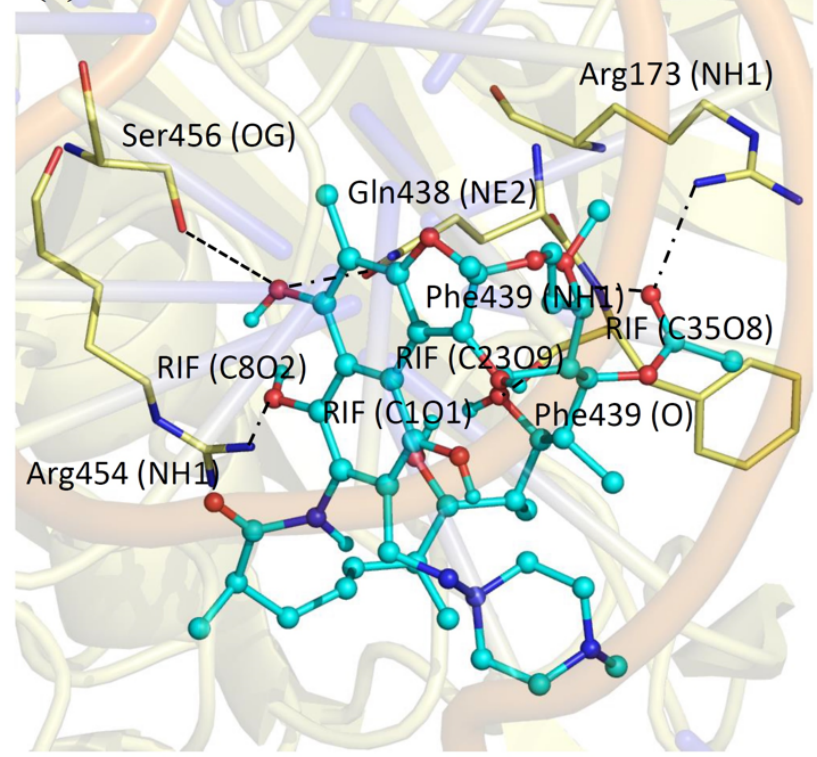

(b)
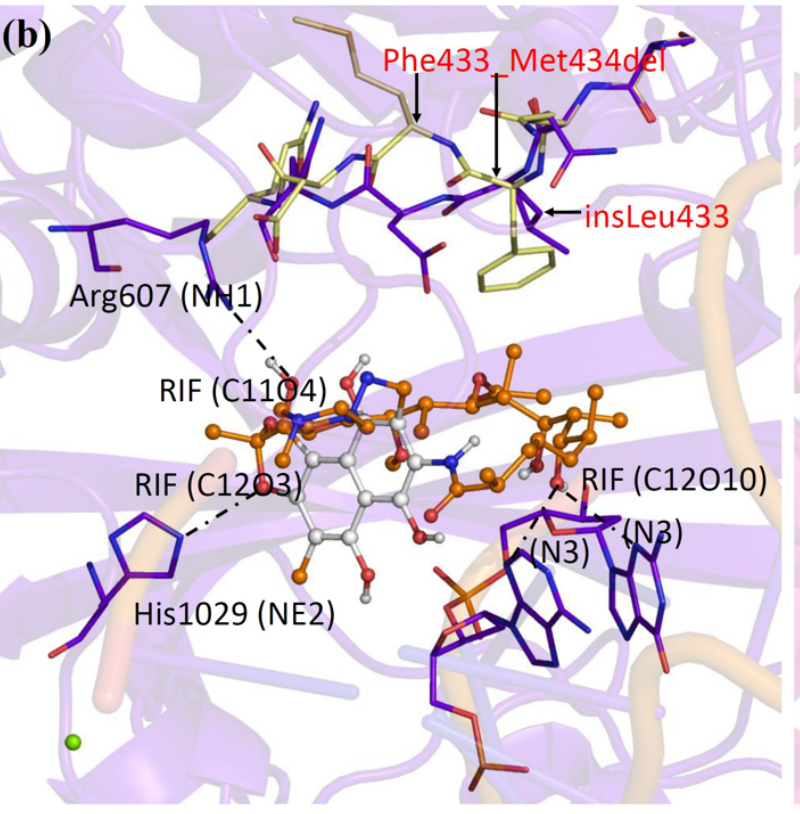

(c)

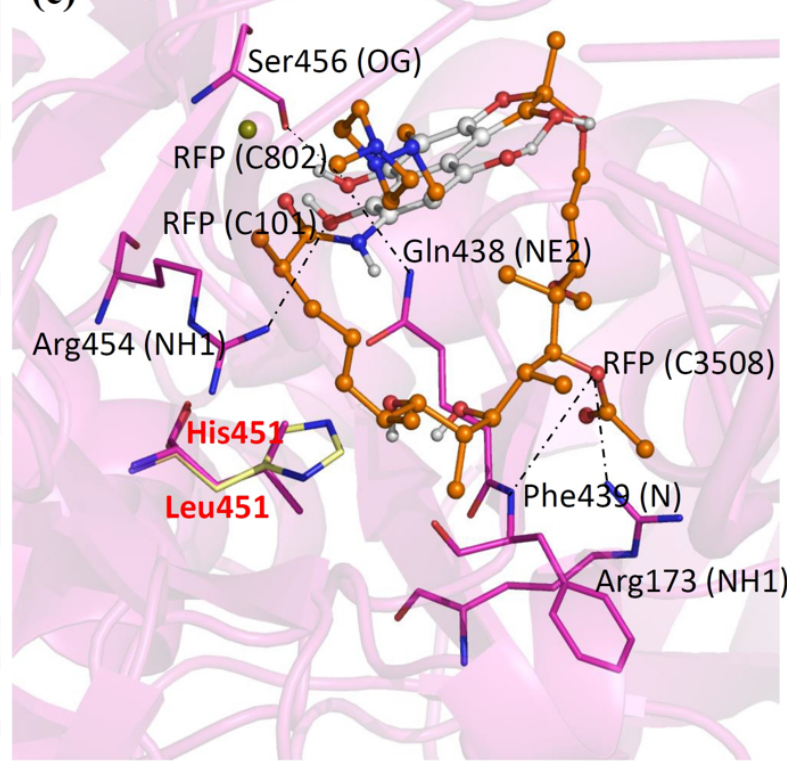

Figure 4. Hydrogen bond interactions between RIF inhibitor and active site residues in wild type and mutant RpoB structures. (a) Wild type structure. (b) Deletion of variant, Phe433_Met434delinsLeu in MTBR1/09 strain. (c) Missense variants, Lys37Arg and His445Leu in MTBR3/09 strain. RIF inhibitor in wild type and mutant are in cyan and orange, respectively. Wild type residues are in yellow. Mutant residues are in purple and magenta. 
(a)

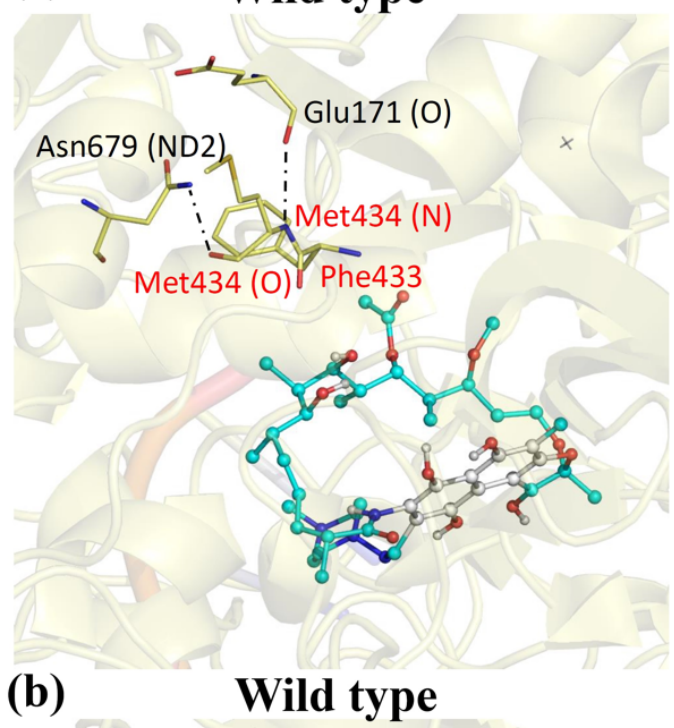

(b)

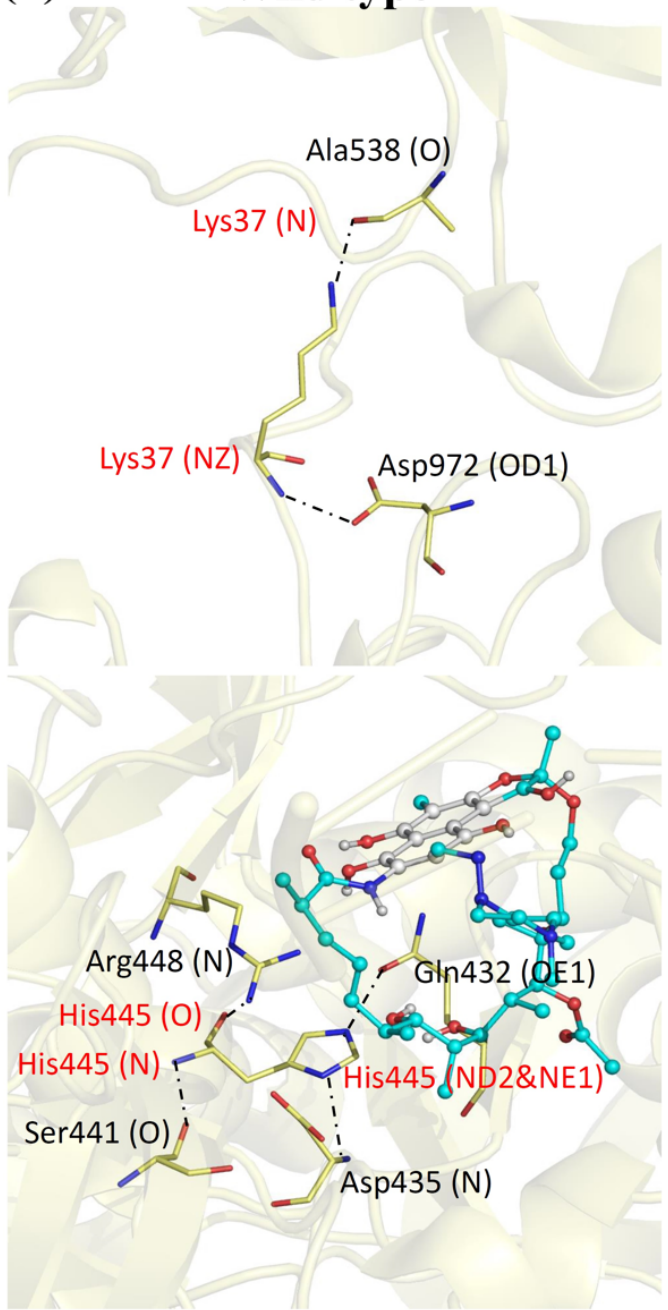

Mutant

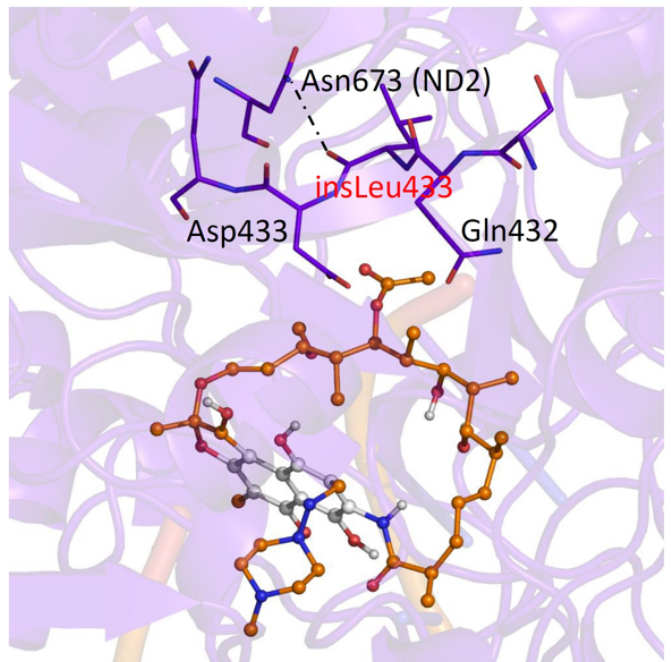

Mutant

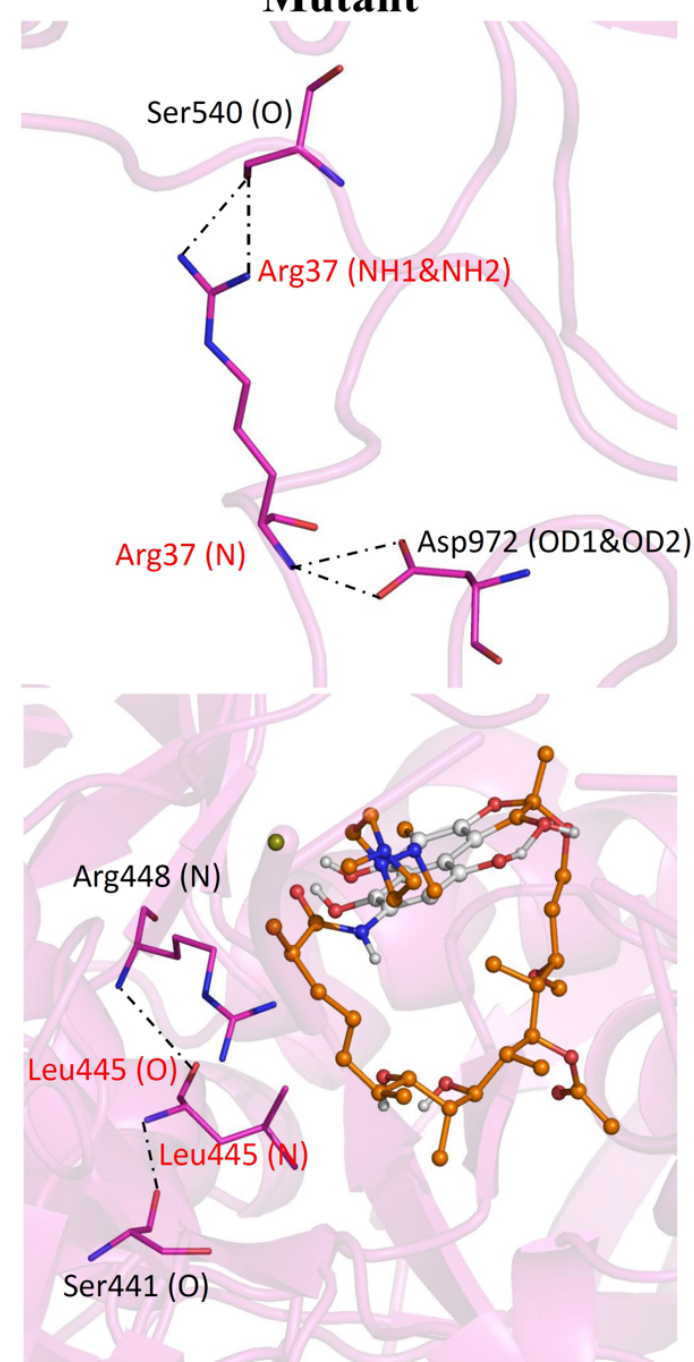

Figure 5. Hydrogen bond interactions between wild type, mutant residues, and neighboring residues. (a) Deletion of variant, Phe433_Met434delinsLeu in MTBR1/09 strain. (b) Missense variants, Lys37Arg and His445Leu in MTBR3/09 strain. RIF inhibitor in wild type and mutant are in cyan and orange, respectively. Wild type residues are in yellow. Mutant residues are in purple and magenta. 
The effect of the new missense variant, Lys37Arg along with the reported variant, His445Leu on the MTBR3/09 strain causes RIF to bind to the RIF binding site with a different conformation, but still may well preserve the interactions between RIF and RpoB protein. Although the residue of Lys37Arg is located far from the RIF binding site, but not to the residue of His445Leu, which is located near to the RIF binding site. Previous studies revealed the specific variants at residue His445 involve in drug resistance and also can maintain the RIF binding to the RpoB protein (Kumar and Lingaraja, 2014; Miotto et al., 2018). Therefore, based on the outcome, it can be postulated that both variants in MTB3R/09 strain might involve in the RIF resistance.

Previous studies describe majority of the variants are located at RRDR of $r p o B$ gene, which spanning codons 433-458 (codon 507-533, Escherichia coli numbering system) (Singh et al., 2017; Zaw et al., 2018). About more than $96 \%$ of RIF-resistant strains have variants in this region (Almeida da Silva and Palomino, 2011; Kumar and Lingaraja, 2014; Singh et al., 2017; Zaw et al., 2018). The non-synonymous are the more common variants rather than insertion, deletion and frameshift (Zaw et al., 2018). However, several studies reveal that the insertion and deletion variants are also responsible to the RIF resistance (Mani et al., 2001; Sharma and Madan, 2014; Miotto et al., 2018). In conclusion, by considering the location of the new deletion and reported missense variants obtained from this study, the resistance of RIF towards all strains from the DST, and the docking results, it can be postulated that all variants were responsible to cause the RIF resistance. In addition, molecular dynamics simulation is suggested to be carried out to prove the stability of RIF binds to the mutant models and relation with the resistance.

\section{ACKNOWLEDGEMENTS}

This study was funded by the Ministry of Health (MOH) of Malaysia (NMRR-18-728-41333). The authors are indebted to the Director General of Health, Malaysia and Director of Institute for Medical Research for permission to publish this article. The authors would like to extent the gratitude to National Public Health Laboratory, Malaysia for the bacterial cultures. The authors also would like to extent the gratitude to Nurul Akma Mohd Hassan, Valentinus H. Seradja and Nur Azieyati Abdullah from Bacteriology Unit for their excellent technical assistance.

\section{CONFLICT OF INTERESTS}

The authors declare that they have no competing interest.

\section{REFERENCES}

Al-Mutairi, N. M., Ahmad, S., Mokaddas, E., Eldeen, H. S., \& Joseph, S. 2019. Occurrence of disputed rpoB mutations among Mycobacterium tuberculosis isolates phenotypically susceptible to rifampicin in a country with a low incidence of multidrug-resistant tuberculosis. BMC Infectious Diseases 19 (1): 1-9.

Almeida da Silva, P. E., \& Palomino, J. C. 2011. Molecular basis and mechanisms of drug resistance in Mycobacterium tuberculosis: Classical and new drugs. Journal of Antimicrobial Chemotherapy 66: 1417-1430.

Benkert, P., Biasini, M., \& Schwede, T. 2011. Toward the estimation of the absolute quality of individual protein structure models. Bioinformatics 27 (3): 343-350.

Bolton, E. E., Wang, Y., Thiessen, P. A., \& Bryant, S. H. 2008. PubChem: Integrated Platform of Small Molecules and Biological Activities. Chapter 12 In Wheeler RA and Spellmeyer DC, eds. Annual Reports in Computational Chemistry (Vol 4: pp. 217-241). Oxford, UK: Elsevier.

Borukhov, S. \& Nudler, E. 2003. RNA polymerase holoenzyme: Structure, function and biological implications. Current Opinion in Microbiology 6 (2): 93-100.

Campbell, E. A., Korzheva, N., Mustaev, A., Murakami, K., Nair, S., Goldfarb, A., \& Darst, S. A. 2001. Structural mechanism for rifampicin inhibition of bacterial RNA polymerase. Cell 104 (6): 901-912.

Chernyaeva, E. N., Shulgina, M. V, Rotkevich, M. S., Dobrynin, P. V, Simonov, S. A., Shitikov, E. A., Ischenko, D. S., Karpova, I. Y., Kostryukova, E. S., Ilina, E. N., Govorun, V. M., Zhuravlev, V. Y., Manicheva, O. A., Yablonsky, P. K., Isaeva, Y. D., Nosova, E. Y., Mokrousov, I. V., Vyazovaya, A. A., Narvskaya, O. V., Lapidus, A. L., \& O’Brien, S. J. 2014. Genome-wide Mycobacterium tuberculosis variation (GMTV) database: A new tool for integrating sequence variations and epidemiology. BMC Genomics 15 (308): 1-8.

Cole, S. T. 1996. Rifamycin resistance in mycobacteria. Research in Microbiology 147 (1-2): 48-52.

Colovos, C. \& Yeates, T. O. 1993. Verification of protein structures: Patterns of nonbonded atomic interactions. Protein Science 2: 1511-1519.

De Azevedo, W. F. Jr. 2010. MolDock Applied to Structure-Based Virtual Screening. Current Drug Targets 11 (3): 327-334.

DeLano, W. L. 2002. The PyMOL molecular graphics system. DeLano Scientific, San Carlos, CA, USA.

Fakhruzzaman, M. N., Abidin, N. Z., Aziz, Z. A., Lim, W. F., 
Richard, J. J., Noorliza, M. N., Hani, M. H., Norhayati, R., Zamzurina, A. B., Zuraina, F. M. Y., Hisyam, M. J., Teh, L. K., Norazmi, N. M., \& Zaki, M. S. 2019. Diversified lineages and drug-resistance profiles of clinical isolates of Mycobacterium tuberculosis complex in Malaysia. International Journal of Mycobacteriology 8: 320-328.

Gerald, M., Carolyn, N., \& Willy, S. 2014. Rifampicin resistance mutations in the $81 \mathrm{bp}$ RRDR of $r p o B$ gene in Mycobacterium tuberculosis clinical isolates using Xpert ${ }^{\circledR}$ MTB/RIF in Kampala, Uganda: A retrospective study. BMC Infectious Diseases 14 (481): 1-5.

Goldstein, B. P. 2014. Resistance to rifampicin: A review. Journal of Antibiotics 67 (9): 625-630.

Guha, R., Howard, M. T., Hutchison, G. R., Murray-Rust, P., Rzepa, H. S., Steinbeck, C., Wegner, J., \& Willighagen, E. L. 2006. The blue obelisks-interoperability in chemical informatics. Journal of Chemical Information and Modeling 46 (3): 991-998.

Issa, R., Seradja, V. H., Abdullah, M. K. H., \& Abdul, H. 2016. Annotated whole-genome shotgun sequence of Mycobacterium tuberculosis MTBR2/09 isolated from a sputum sample in Malaysia. Genome Announcement 4 (3): e00517-16.

Jani, J., Mustapha, Z. A., Jamal, N. B., Stanis, C. S., Ling, C. K., Avoi, R., Tha, N. O., Gantul, V., Mori, D., \& Ahmed, K. 2019. Whole genome sequencing data and analysis of a rifampicin-resistant Mycobacterium tuberculosis strain SBH162 from Sabah, Malaysia. Data in Brief 26 (104445): 1-5.

Kumar, S. \& Lingaraja, J. 2014. Understanding rifampicin resistance in tuberculosis through a computational approach. Genomics Informatics 12 (4): 276-282.

Kumar, S., Stecher, G., \& Tamura, K. 2016. MEGA7: Molecular Evolutionary Genetics Analysis version 7.0 for bigger datasets. Molecular Biology and Evolution 33 (7): 1870-1874.

Laskowski, R. A., MacArthur, M. W., \& Thornton, J. M. 2012. PROCHECK: Validation of protein-structure coordinates. In: International Tables for Crystallography (Vol. F, ch. 21.4. pp. 684-687). Crystallography of Biological Macromolecules Kluwer Academic Publishers The Netherlands.

Lin, W., Mandal, S., Degen, D., Liu, Y., Ebright, Y. W., Li, S., Feng, Y., Zhang, Y., Mandal, S., Jiang, Y., Liu, S., Gigliotti, M., Talaue, M., Connell, N., Das, K., Arnold, E., \& Ebright, R. H. 2017. Structural basis of Mycobacterium tuberculosis transcription and transcription inhibition. Molecular Cell 66 (2): 169-179.

Mackerell, A. D., Feig, M., \& Brooks III, C. L. 2004. Extending the treatment of backbone energetics in protein force fields: Limitations of gas-phase quantum mechanics in reproducing protein conformational distributions in molecular dynamics simulation. Journal of Computational Chemistry 25 (11): 1400-1415.

Mani, C., Selvakumar, N., Narayanan, S., \& Narayanan, P. R. 2001. Mutations in the $r p o B$ gene of multidrug-resistant Mycobacterium tuberculosis clinical isolates from India. Journal of Clinical Microbiology 39 (8): 2987-2990.

Miotto, P., Cabibbe, A. M., Borroni, E., Degano, M., \& Cirillo, D. M. 2018. Role of disputed mutations in the $r p o B$ gene in interpretation of automated liquid MGIT culture results for rifampin susceptibility testing of Mycobacterium tuberculosis. Journal of Clinical Microbiology 56: 01599-17.

Nusrath, U. A., Hassan, S., Indira Kumari, V., Revathy, R., \& Hanna, L. E. 2016. Insights into RpoB clinical mutants in mediating rifampicin resistance in Mycobacterium tuberculosis. Journal of Molecular Graphics and Modelling 67: 20-32.

Nusrath, U. A. \& Hanna, L. E. 2017. Molecular mechanisms of action, resistance, detection to the first-line anti tuberculosis drugs: Rifampicin and pyrazinamide in the post whole genome sequencing era. Tuberculosis 105: 96-107.

Pang, Y., Lu, J., Wang, Y., Song, Y., Wang, S., \& Zhao, Y. 2013.
Study of the rifampin monoresistance mechanism in Mycobacterium tuberculosis. Antimicrobial Agents and Chemotherapy 57 (2): 893-900.

Peterson, S. M., Thompson, J. A., Ufkin, M. L., Sathyanarayana, P., Liaw, L., \& Congdon, C. B. 2014. Common features of microRNA target prediction tools. Frontiers in Genetics 5: 110.

Philip, N., Rodrigues, K. F., William, T., \& John, D. V. 2016. Whole genome sequencing of Mycobacterium tuberculosis SB24 isolated from Sabah, Malaysia. Genomics Data 9: 137-139.

Prakash, R., Gupta, R., Katoch, V. M., \& Tiwari, P. K. 2017. Molecular modelling and docking analysis of kat $G$ and $r p o B$ gene in MDR-TB isolates from North Central Indian population. Journal of Infection and Public Health 10 (5): 593599.

Sanner, F. M. 1999. Python: A programming language for software integration and development. Journal of Molecular Graphics and Modelling 17 (1): 57-61.

Sekhar, P. N., Kishor, P. B. K., Reddy, V. C. K., Kumar, E. P., Anitha, A. M. R. K., \& Reddy, L. A. 2008. Comparative modeling and docking studies of Mycobacterium tuberculosis H37RV rpoB protein. Journal of Molecular Design 7 (1): 12-29.

Sharma, S. \& Madan, M. 2014. Detection of mutations in rpob gene of clinically isolated $M$. Tuberculosis by DNA sequencing. Mycobacterial Diseases 4 (4): 1-5.

Sievers, F., Wilm, A., Dineen, D., Gibson, T. J., Karplus, K., Li, W., Lopez, R., McWilliam, H., Remmert, M., Soding, J., Thompson J. D., \& Higgins D. G. 2011. Fast, scalable generation of high-quality protein multiple sequence alignments using Clustal Omega. Molecular Systems Biology 7: 539 .

Singh, A., Grover, S., Sinha, S., Das, M., Somvanshi, P., \& Grover, A. 2017. Mechanistic principles behind molecular mechanism of rifampicin resistance in mutant RNA polymerase beta subunit of Mycobacterium tuberculosis. Journal of Cellular Biochemistry 118: 4594-4606.

Trott, O. \& Olson, A. J. 2010. AutoDock Vina: Improving the speed and accuracy of docking with a new scoring function, efficient optimization and multithreading. Journal of Computational Chemistry 31 (2): 455-461.

Waterhouse, A., Bertoni, M., Bienert, S., Studer, G., Tauriello, G., Gumienny, R., Heer, F. T., de Beer, T. A. P., Rempfer, C., Bordoli, L., Lepore, R., \& Schwede, T. 2018. SWISSMODEL: Homology modelling of protein structures and complexes. Nucleic Acids Research 46: 296-303.

Wiederstein, M. \& Sippl, M. J. 2007. ProSA-web: Interactive web service for the recognition of errors in three-dimensional structures of proteins. Nucleic Acids Research 35(Web Server issue): W407-W410.

World Health Organization. 2019. WHO Global Report, Global Tuberculosis Report 2019. Geneva. World Health Organization; 2019.

Zaw, M. T., Emran, N. A., \& Lin, Z. 2018. Mutations inside rifampicin-resistance determining region of $r p o B$ gene associated with rifampicin-resistance in Mycobacterium tuberculosis. Journal of Infection and Public Health 11: 605-610.

Zhang, G., Campbell, E. A., Minakhin, L., Richter, C., Severinov, K., \& Darst, S. A. 1999. Crystal structure of Thermus aquaticus core RNA polymerase at 3.3 A resolution. Cell 98 (6): 811-824.

Zhang, H., Li, D., Zhao, L., Fleming, J., Lin, N., Wang, T., Zhou, Y., Zhu, Y., Gao, Y., Wang, T., Wang, S., Huang, Y., Wang, M., Zhong, Q., Zhou, L., Chen, T., Zhou, J., Yang, R., Zhu, G., Hang, H., Zhang, J., Li, F., Wan, K., Wang, J., Zhang, X., \& Bi, L. 2013. Genome sequencing of 161 Mycobacterium tuberculosis isolates from China identifies genes and intergenic regions associated with drug resistance. Nature Genetics 45 (10): 1255-1260. 